\title{
AN ANALOGY BETWEEN SIMULATED AND ACTUAL CLUSTERS OF DIFFERENT KINDS
}

\section{G. Paá1}

A study of clusters is presented by means of the function $\mathrm{N}_{\mathrm{m}}(\theta)$, i.e. the total number $\mathrm{N}$ of members heavier than a limiting mass $\mathrm{m}$, as a function of the limiting projected angular distance from the centre $\theta$. As a rule such a function is found to possess a we11-defined maximum change of slope - an apparent "deflection" - if the limiting mass is large enough (see Figure $1 \mathrm{a}-\mathrm{g}$ ). Some possible definitions of this deflection and the corresponding special "core radius" of the cluster

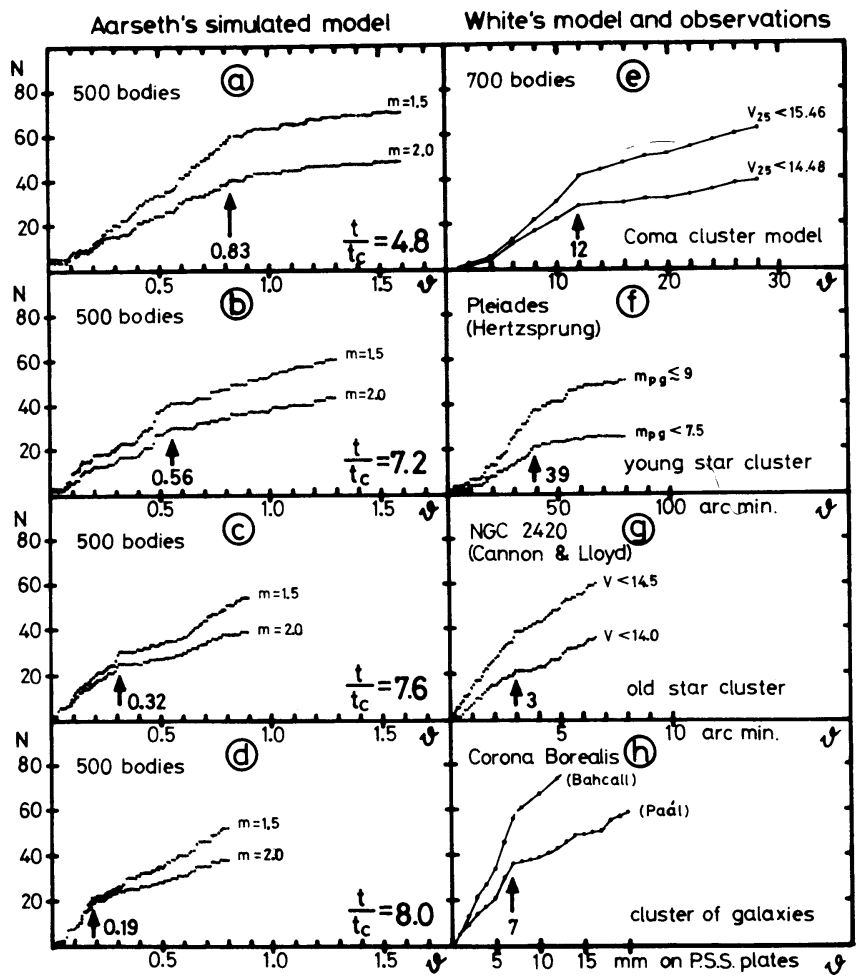

Further diagrams of observed clusters: Astrofizika 7,pp. 438, 445.

Figure 1. are described elsewhere (Astrofizika, 7, 435, 1971). The typical occurrence and rather regular behaviour (systematic tempora1 variations) of this deflection in a sample of 17 configurations simulated by S.J. Aarseth and S.D.M. White for different conditions prove this feature to be a natura1 consequence of the gravitational interaction of many bodies in a statistical sense; it is apparently a characteristic of core formation and the evolution of clusters. In the case of computer data the existence of such deflections cannot possibly be due to any observational error or uncertainty or erroneous personal judgement. The corresponding diagrams, $\mathrm{N}_{\mathrm{m}}(\theta)$ for clusters of galaxies (see also 1oc. cit.) are found to be practically indistinguishable from those of star clusters and simulated systems. They show the very same type of deflection, the same remarkably small dependence on the choice of the limiting mass, that of the "centre", the field correction etc. As simulations give just the same diagrams as have been obtained observationally it is plausible 
to regard also these observational findings as dynamically necessary and not chance coincidences, unless the actual clusters of galaxies are stabilized by some continuously distributed intergalactic "hidden matter" instead of the masses of observable discrete bodies themselves. However one can hardly accept a radically different explanation for the apparently identical phenomenon in the case of systems of galaxies on the one hand and those of stars on the other. Estimates of the crossing time $t_{c}$ of the systems together with morphological arguments and numerical experiments indicate that actual "rich" clusters of galaxies might typically be near a particular epoch of very rapid systematic cosmological evolution. A considerable decrease of the aforementioned "core radii" (a transition between two nearly constant values) with the other characteristic sizes of the systems almost unchanged (Figure $1 \mathrm{a}-\mathrm{d}$ ). The corresponding phenomena were detected earlier observationally and consequently these empirical data, together with their consequences, now seem to be once again corroborated (see e.g. Astron. Nachrichten, 297, $311,1976)$. These remarkable analogies suggest that a similar mechanism is responsible for the formation and evolution of conspicuous cores of clusters and a similar solution of the somewhat controversial situation with regard to mass segregation both for clusters of galaxies and open star clusters (e.g. by properly choosing the initial conditions).

\section{STRUCTURES AND NUMBER-DENSITY DISTRIBUTIONS} IN CLUSTERS OF GALAXIES

\section{F. W. Baier}

I would like to present some preliminary results of an investigation of 50 clusters of galaxies. For studying the clusters, Schmidt plates taken with the 2-metre universal telescope at Tautenburg and the prints of the Palomar Sky Survey were used. For all the clusters we determined isopleths to obtain detailed information about the distribution of galaxies. We have found well isolated clusters, double clusters, multiple systems and rather complicated structures.

Because we cannot say anything about physical connections between the various groupings in the cluster regions under investigation, we certainly have to expect a high percentage of random projections of independent clusters. But we cannot exclude the possibility of physical connections between the various groupings in those cases in which we observe an irregular but well isolated structure.

For the isolated clusters we calculated projected radial numberdensity distributions and radial cumulative distributions. From the latter we found the total populations and dimensions of the clusters. But these quantities have to be considered upper limits for those clusters which are not well isolated because neighbouring clusters produce secondary maxima in the radial number-density distribution and increase both the total number of galaxies and the distance from the centre to the apparent border of the cluster. 